\title{
ОСНОВНЫЕ ПРИНЦИПЫ ОСУЩЕСТВЛЕНИЯ КОНТРОЛЬНО-АУДИТОРСКИХ МЕРОПРИЯТИЙ ПРИ УЧЕТЕ ДОХОДОВ И РАСХОДОВ В АВТОНОМНЫХ УЧРЕЖДЕНИЯХ
}

\section{BASIC PRINCIPLES OF IMPLEMENTATION OF CONTROL AND AUDITING MEASURES WHEN RECOGNIZING INCOME AND EXPENDITURE IN AUTONOMOUS INSTITUTIONS}

\section{T. Turishcheva}

Summary. The socio-economic development of any country is a multifaceted process that includes economic growth and an increase in the level and quality of life of the population. First of all, it depends on a balanced budget policy, which includes legislatively defined measures aimed at the formation of the state's monetary fund, and provides for the rational distribution of budgetary resources in all spheres of society. An integral element of ensuring the targeted and rational use of budget funds is a timely and thorough audit and control of the income and expenses of organizations supported by public funds, including autonomous institutions, in compliance with the basic principles aimed at ensuring the legality and effectiveness of the actions of all participants in the budget process. Given the above, the purpose of the article is to formulate and clarify the content of the principles of budgetary control and audit of revenues and expenditures of autonomous institutions. In the course of the study, the author clarified the general principles of audit and control of income and expenses of autonomous institutions, as well as formulated specific principles, taking into account the peculiarities of budget execution, which together should contribute to timely and high-quality management decisions in the budget process based on complete, relevant and systematized information.

Keywords: autonomous institution, income, expenses, control, audit, principles, legality, efficiency, rationality, consistency.

\section{Ввеление}

Постановка проблемы в общем виде и ее связь с важными научными и практическими задачами

юджетная система играет важную роль в экономике любого государства и значительным образом влияет на внутренний и внешний баланс хозяйственной системы, динамику развития экономических процессов и благосостояние населения в целом [1].

\author{
Турищева Татьяна Борисовна \\ К.э.н., дочент, РЭА им. Г.В. Плеханова; Финансовый \\ университет при Правительстве РФ \\ ttb2812@mail.ru
}

Аннотация. Социально-экономическое развитие любой страны представляет собой многоплановый процесс, охватывающий экономический рост, повышение уровня и качества жизни населения. Достижение обозначенных целей, прежде всего, зависит от взвешенной бюджетной политики, которая включает законодательно определенные меры, направленные на формирование денежного фонда государства, и предусматривает рациональное распределение бюджетных ресурсов для обеспечения всех сфер жизни общества. Неотъемлемым элементом целевого и рационального использования бюджетных средств является проведение своевременных и основательных контрольно-аудиторских мероприятий при учете доходов и расходов организаций, содержащихся за счет государственных средств, в том числе автономных учреждений, с соблюдением основных принципов, направленных на обеспечение законности и эффективности действий всех участников бюджетного процесса. В контексте вышеизложенного, цель статьи заключается в формулировке и уточнении содержания принципов осуществления государственного финансового контроля и аудита учета доходов и расходов автономных учреждений. В процессе исследования автором уточнены общие принципы осуществления контрольно-аудиторских мероприятий по учету доходов и расходов автономных учреждений, а также сформулированы специфические, характерные принципы, базирующиеся на особенностях выполнения сметы, которые в совокупности должны способствовать своевременному и качественному принятию управленческих решений в бюджетном процессе на основе полной, актуальной и систематизированной информации.

Ключевые слова: автономное учреждение, доходы, расходы, контроль, аудит, принципы, законность, эффективность, рациональность, системность.

Развитие бюджетных отношений в России на сегодняшний день связано с радикальными преобразованиями, происходящими в связи с реформированием бюджетного законодательства, реализацией плана по децентрализации властных полномочий, построением современного, правового, демократического государства, попытками создать новую архитектонику институциональной среды общественного развития. Для достижения указанных целей в стране осуществляются изменения, касающиеся определения новых 
правовых рамок формирования бюджетной системы и воспроизводства бюджетного процесса, модернизации механизма аккумулирования и распределения централизованных фондов денежных средств, введения так называемых «партнерских» отношений между государством и обществом.

Вместе с тем следует отметить, что развитие и реформирование отношений в сфере бюджетной деятельности требует четкого контроля и надзора, поскольку эта сфера общественных отношений является потенциально конфликтной в любом государстве. Органы власти и правительственные структуры не могут реализовать свою внешнюю и внутреннюю политику, обеспечить выполнение социально-экономических программ, не имея финансовых ресурсов, а также, не осуществляя соответствующий контроль за законным их формированием и расходованием [2].

Учитывая это, приобретает критически важное значение система аудита и контроля в бюджетной сфере, основные задачи которой заключаются в выявлении бюджетных преступлений, применении мер воздействия на нарушителей бюджетного законодательства, предотвращении злоупотреблений и незаконного расходования средств на всех стадиях бюджетного процесса. Особого внимания в указанных условиях заслуживает система аудита и контроля за наличием и движением бюджетных и внебюджетных средств автономных учреждений.

Наиболее специфическими объектами аудита и контроля в автономных учреждениях являются доходы и расходы, поскольку именно их учет представляется самым сложным и ответственным, требующим детальной проработки и четкой регламентации всех процедур. Целью контрольно-аудиторских мероприятий при учете доходов и расходов в автономных учреждениях является изучение бухгалтерской и финансовой информации по отдельным статьям расходов, анализ остатков на бухгалтерских счетах, проверка их соответствия утверждённой смете и выделяемым ассигнованиям, исследование правильности выполнения бюджетных процедур, проверка точности арифметических расчетов, оценки бюджетных рисков и т.д.

Очевидно, что усовершенствование действующих бюджетных отношений в стране, качественное и эффективное проведение реформ требует четких, адекватных современным требованиям и реалиям, понятных и обоснованных принципов осуществления государственного финансового контроля в целом, а также доходов и расходов учреждений, в частности. Определение принципов контрольно-аудиторских мероприятий имеет важное значение для формирования актуальной концепции бюджетного контроля и надзо- ра, определения наиболее действенных и результативных инструментов осуществления контрольных мероприятий в деятельности автономных учреждений.

Итак, принимая во внимание тот факт, что очерченная выше проблема является очень актуальной и значимой, необходимо осуществлять поиск путей ее решения в научной и практической плоскостях, чему и посвящена данная статья.

\section{Анализ последних исследований и публикаций, в которых рассматривались аспекты этой проблемы и на которых обосновывается автор; выделение неразрешенных раньше частей общей проблемы}

Научную проблему, которая заключается в определении состава и формализации содержания принципов государственного финансового контроля, в том числе бюджетного, в своих трудах исследовали: Felício, Teresa; Samagaio, António; Rodrigues, Ricardo [3], Di Francesco, Michael; Alford, John [4], Hay, David C.; Cordery, Carolyn J. [5], Прокопович А.А. [6], Душакова Л.А., Коломиец К.А. [7].

Детальному изучению особенностей учета доходов и расходов автономных учреждений уделяли внимание: Васильченко А.А. [8], Скрипкина О.В., Минашкина А.Н. [9], van Helden, Jan; Reichard, Christoph [10], Bracci, Enrico; Tallaki, Mouhcine [11], Ипатова A.B. [12].

Такие ученые как Выжитович А.М., Скорлыгина Е.A. [13], Абрамова Е.В., Богатырева O.B. [14], Rana, Tarek; Wickramasinghe, Danture; Bracci, Enrico [15], Vallentin, Steen; Thygesen, Niels [16], Paterson, Audrey S.; Changwony, Fredrick; Miller, Peter B. [17] акцентируют внимание на формах осуществления контроля и аудита в автономных учреждениях, недостатках его нормативно-правового обеспечения, методике проведения и других специфических вопросах.

Несмотря на основательность проработки исследуемой проблематики в научных трудах отечественных и зарубежных авторов, справедливо признать факт наличия нерешенных проблемных аспектов. Так, содержательное наполнение принципов государственного финансового контроля и аудита, которое можно встретить в современных публикациях, является очень широким и разнонаправленным, поскольку некоторые ученые рассматривают контроль как функцию государственного управления, а другие считают, что он является одним из видов государственного контроля. Это в свою очередь приводит к неоднозначной трактовке и вариативности применения норм, которыми регулируются соответствующие отношения и обеспечивается эффективность такого явления, как 


\begin{tabular}{|c|c|c|}
\hline $\begin{array}{c}\text { Деятельность аудиторской } \\
\text { группы и осуществляемые } \\
\text { контрольные меры должны } \\
\text { базироваться только на } \\
\text { четких законодательных } \\
\text { нормах и требованиях }\end{array}$ & $\begin{array}{c}\text { Осуществляемые } \\
\text { контрольные меры должны } \\
\text { обеспечивать объективную } \\
\text { оценку изучаемой области } \\
\text { и деятельности учреждения } \\
\text { в целом }\end{array}$ & $\begin{array}{c}\text { Задачи и функции } \\
\text { контроля и аудита должны } \\
\text { быть четко разделены и } \\
\text { регламентированы }\end{array}$ \\
\hline Законность & Объективность & $\begin{array}{c}\text { Распределение } \\
\text { контрольных полномочий }\end{array}$ \\
\hline
\end{tabular}

\section{ПРИНЦИПЫ БЮДЖЕТНОГО КОНТРОЛЯ}

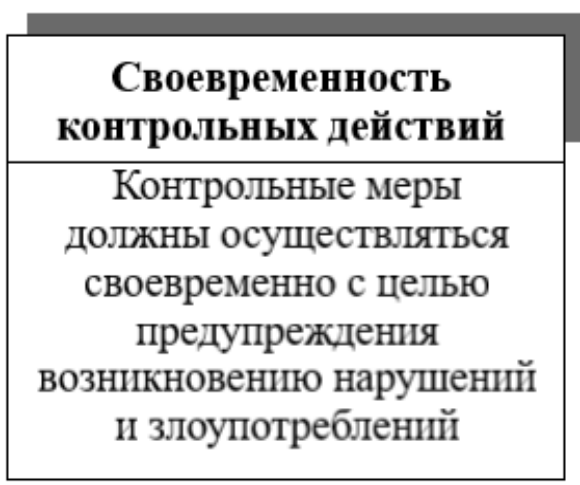
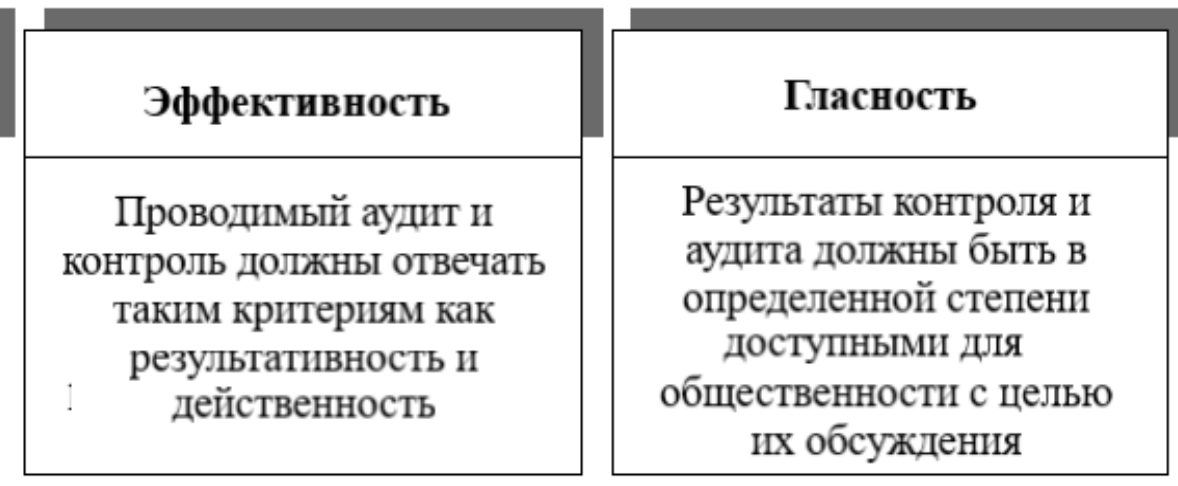

Рис. 1. Общие принципы аудита и контроля учета доходов и расходов автономных учреждений

контроль учета доходов и расходов автономных учреждений.

Учитывая указанное, считаем целесообразным расширить масштабы исследуемой научной проблемы и внести ясность в ее некоторые ключевые аспекты.

\section{Мето ология}

\section{Формирование целей статьи}

Цель статьи заключается в формулировке и уточнении содержания принципов осуществления контрольно-аудиторских мероприятий при учете доходов и расходов в автономных учреждениях.

\section{Используемые в исследовании методы, методики и технологии}

Методологическую основу исследования составляют общенаучные и специальные методы познания экономических явлений и механизмов осуществления государственного финансового контроля. Основные научные результаты исследования были получены на основе применения следующих методов: систем- но-структурный; логическое обобщение и абстрагирование; абстрактная логика и сравнение в сочетании с системным подходом.

\section{Результаты}

\section{Изложение основного материала исследования с полным обоснованием полученных научных результатов}

Значение принципов государственного финансового контроля трудно переоценить, поскольку они консолидируют нормы и институты осуществления контрольной и ревизионной деятельности, наделяют их целостностью; являются исходными положениями или научными мыслями при интерпретации процессуальных действий; подчеркивают общность и специфику бюджетного контроля по сравнению с другими видами надзорной деятельности.

Характеризуя принципы осуществления контрольно-аудиторских мероприятий при учете доходов и расходов в автономных учреждениях, некоторые ученые зачастую отождествляют их с общими принципами финансового контроля, которые имеют свои особенности 
проявления в сфере государственного финансового контроля и описывают их как основные требования, зафиксированные в нормативно-правовых актах, а также правила, которые не нашли своего правового закрепления, но получили всеобщее признание в процессе организации финансового контроля и деятельности субъектов, наделенных контрольными полномочиями или правами. Детальное описание этих общих принципов представлено на рис. 1.

По мнению автора, указанная позиция в некоторой степени является обоснованной, поскольку контрольно-аудиторские мероприятия при учете доходов и расходов в автономных учреждениях представляют собой неотъемлемую часть общего государственного финансового контроля, что соответственно обусловливает необходимость придерживаться единых принципов, выполнение которых должно быть первоочередным при организации и осуществлении любых контрольных мероприятий.

Вместе с тем, такая интерпретация принципов, по нашему мнению, является достаточно широкой и подчеркивает якобы их производный характер от общего государственного контроля. Не в пользу данного подхода также свидетельствует тот факт, что эти два направления контроля находятся на разных уровнях обобщения, выполняют очень похожие, но все же разные функции и имеют разное социальное значение. Кроме того, такая позиция не учитывает специфики доходов и расходов автономных учреждений, на чем уже было акцентировано внимание ранее.

Поэтому, соглашаясь с тем, что фундаментальные установки и правила осуществления бюджетного контроля должны быть общими для любой плоскости предлагаем приведенные на рис. 1 принципы дополнить следующими:

- принцип системности означает, что аудит и контроль учета доходов и расходов должны быть интегрированы в общую систему управления автономным учреждением и обеспечивать планомерную реализацию его основных задач в пределах выделенных бюджетных ассигнований;

- принцип комплексности предполагает, что в процессе осуществления контрольно-аудиторских мероприятий необходимо принимать во внимание результаты использования бюджетных средств учреждением в предыдущие периоды, а также учитывать прогноз основных показателей его деятельности и общий макроэкономический прогноз по стране. Контроль учета доходов и расходов автономных учреждений во взаимосвязи с макроэкономическим прогнозированием и результатами прошлых периодов позволит правильно оценить возможность полного и стабильного финансирования предполагаемых мероприятий и последствия их реализации;

- принцип реалистичности заключается в подготовке адекватной оценки относительно потребностей в финансировании основных функций и задач автономного учреждения и направлении бюджетных средств на мероприятия, реализация которых позволит получить конкретный эффект.

Использование в практической деятельности принципов осуществления контрольно-аудиторских мероприятий при учете доходов и расходов в автономных учреждениях, в рамках которых будет акцентироваться внимание как на современном состоянии общественных отношений, так и на перспективах их развития, позволит сформировать относительно устойчивую систему специальных (или точнее - «внутренних») принципов контроля.

\section{Обсужление}

\section{Сравнение полученных результатов \\ с результатами в других исследованиях}

Как уже отмечалось ранее, в силу объективных обстоятельств общие принципы не могут отразить специфику исследуемой плоскости бюджетного контроля, но в тоже время они являются теми исходными концептуальными принципами, которые сочетают части в единое целое. Поэтому логичным дополнением общих принципов являются частные, более предметные и конкретные.

Рассматривая более детально принципы осуществления контрольно-аудиторских мероприятий при учете доходов и расходов в автономных учреждениях, необходимо отметить, что их формализация и уточнение в отличие, например, от контроля за целевым использованием бюджетных средств, на сегодняшний день развиты недостаточно. Объясняется это в большинстве свое внутренними факторами, а именно ориентацией учета на составление финансовой отчетности, недостаточным вниманием к вопросам оптимизации использования ресурсов на микроуровне и сложностью разработки адекватных моделей контроля.

Принимая во внимание вышеизложенное, считаем, что основой для формализации специфических, характерных принципов осуществления аудита и контроля за учетом доходов и расходов автономных учреждений могут послужить их ключевые цели и задачи, к числу которых относятся:

- проведение объективной оценки состояния выполнения сметы автономного учреждения; 
- выявление нарушений финансовой и бюджетной дисциплины, которые были допущены в хозяйственно-финансовой деятельности учреждений и касаются соблюдения действующих законодательных и нормативных актов, регламентирующих: повышение эффективности расходования бюджетных средств по видам расходов и их функциональной направленности; правильную кодировку и классификацию полученных доходов; обеспечение экономного использования бюджетных ресурсов, а также сохранности средств и материальных ценностей; разработку мероприятий для ликвидации выявленных нарушений и недостатков; возмещение убытков, причиненных автономному учреждению действиями или бездействием ответственных должностных лиц;

- предотвращение злоупотреблений в дальнейшей работе учреждения.

Итак, в число принципов осуществления контрольно-аудиторских мероприятий при учете доходов и расходов в автономных учреждениях, которые учитывают специфику и проблематику проверяемой предметной плоскости, по мнению автора, следует отнести следующие:

- принцип единства и точности. Как известно, за каждый финансовый год все доходы и расходы должны быть внесены и четко отражены в статьях и регистрах бухгалтерского учета. Соответственно контрольные процедуры должны быть четко ограничены этими рамками и проверять факты осуществления расходов сверх выделенных ассигнований;

- принцип спецификации. Согласно принципу спецификации, каждый вид доходов должен иметь конкретное предполагаемое использование и быть предназначенным для достижения определенной цели, чтобы избежать путаницы между различными ассигнованиями либо когда они санкционированы, либо когда реализованы. В процессе аудита и контроля проверяющие должны быть уверены в том, что санкционированные расходы осуществляются с указанной целью и должным образом отражены во всех регистрах учета. Тот же принцип применим и к доходам, которые могут быть получены из четкой обозначенных и согласованных источников;

- принцип значения. Результат, полученный в процессе аудита, очень важен и актуален для соблюдения бюджетной дисциплины и выполнения автономным учреждением своих функций. Важность может проявляться в различных аспектах, например, финансовом, социальном, экономическом, экологическом и тому подобное.
- принцип надежности: выводы должны быть обоснованы и отражать существенные особенности объекта аудита в соответствии с поставленной целью, а также быть достаточно доказательными;

- принцип рационального объема: программа/ план аудита и контроля должны содержать вопросы/задачи, которые могут быть полностью выполнены в соответствии с утвержденными стандартами, правилами и процедурами.

Указанные принципы не являются статичными и окончательно закрепленными, так как в бюджетном процессе постоянно создаются условия для формирования новых подходов, на основании которых возникают объективные, универсальные, необходимые закономерности взаимоотношений между субъектом и объектом контроля, что приводит к появлению новых принципов.

\section{Выво $\triangle$}

\section{Выводы исследования}

Принципы контроля и аудита - это система элементов, которая составляет фундамент методологической базы контрольных и ревизионных мероприятий. Определение принципов контроля имеет важнейшее значение для формирования современной концепции государственного финансового контроля, для ее нормативно-правового обеспечения и внедрения в практику бюджетного процесса.

В процессе исследования автором уточнены общие принципы осуществления контрольно-аудиторских мероприятий при учете доходов и расходов в автономных учреждениях, а также сформулированы специфические с учетом особенностей данной сферы контроля, которые в совокупности должны способствовать своевременному и качественному принятию управленческих решений в бюджетном процессе на основе достоверной, точной и систематизированной информации.

\section{Перспективы дальнейших изысканий в данном направлении}

С учетом прохождения в сфере государственного финансового контроля реформы, считаем, что в настоящее время необходимо сфокусировать внимание на исследовании принципов стратегической направленности и поэтапности контрольно-аудиторских мероприятий, в том числе в сфере учета доходов и расходов автономных учреждений и, таким образом, развить теоретические основы государственного финансового контроля и обеспечить выполнение этих принципов на практике. 


\section{ЛИТЕРАТУРА}

1. Мельник М.В. Тенденции развития государственного финансового контроля и аудита // Вестник профессионального бухгалтера. 2020. № 4-6 (235237). C. 40-58.

2. Dahan, Momi Budget institutions and government effectiveness // Journal of public budgeting, accounting \& financial management. 2020. Volume 32: Number 2; pp 217-246.

3. Felício, Teresa; Samagaio, António; Rodrigues, Ricardo Adoption of management control systems and performance in public sector organizations // Journal of business research. 2021. Volume 124; pp 593-602.

4. Di Francesco, Michael; Alford, John Balancing budget control and flexibility: the central finance agency as 'responsive regulator' // Public management review. 2017. Volume 19: Issue 7; pp 972-989.

5. Hay, David C.; Cordery, Carolyn J. The future of auditing research in the public sector // Journal of public budgeting, accounting \& financial management. 2020. Volume 33: Number 2; pp 234-242.

6. Прокопович А.А. Эволюция становления и развития государственного финансового контроля в России // Учет и контроль. 2020. № 4 (54). С. 2-9.

7. Душакова Л.А., Коломиец К.А. Оценка состояния государственного финансового контроля и надзора в РФ // Аллея науки. 2020. Т. 2. № 6 (45). С. 494-500.

8. Васильченко А.А. Планирование доходов и расходов автономного учреждения // Теория и практика современной науки. 2020. № 9 (63). С. $52-56$.

9. Скрипкина 0.В., Минашкина А.Н. Внутренняя отчетность автономного учреждения // Управленческий учет. 2018. № 2. С. 76-81.

10. van Helden, Jan; Reichard, Christoph Management control and public sector performance management // Baltic journal of management. 2018. Volume 14: Number 1; pp 158-176.

11. Bracci, Enrico; Tallaki, Mouhcine Resilience capacities and management control systems in public sector organisations // Journal of accounting \& organizational change. 2021. Volume 17: Number 3; pp 332-351.

12. Ипатова А.В. Учет доходов от предпринимательской деятельности в государственных автономных учреждениях // Интеграция наук. 2018. № 4 (19). C. 121-123.

13. Выжитович А.М., Скорлыгина Е.А. Система внутреннего аудита автономного учреждения // Вестник НГУЭу. 2018. № 1. С. 122-130.

14. Абрамова Е.В., Богатырева 0.В. Внутренний финансовый контроль как инструмент построения эффективной системы управления в бюджетной сфере экономики // Фундаментальные исследования. 2017. № 9-1. С. 112-116.

15. Rana, Tarek; Wickramasinghe, Danture; Bracci, Enrico New development: Integrating risk management in management control systems — lessons for public sector managers // Public money \& management. 2019. Volume 39: Issue 2; pp 148-151.

16. Vallentin, Steen; Thygesen, Niels Trust and control in public sector reform: Complementarity and beyond // Journal of trust research. 2017. Volume 7: Issue 2; pp 150-169.

17. Paterson, Audrey S.; Changwony, Fredrick; Miller, Peter B. Accounting control, governance and anti-corruption initiatives in public sector organisations // The British accounting review. 2019. Volume 51: Number 5; pp 189-194.

18. Бедоева 3.Н. Принципы плановости и эффективности осуществления государственного бюджетного контроля // Финансовое право. 2020. № 2. С. 26-29.

19. Мальцев В.А. Принципы государственного финансового контроля и проблемы их реализации в Российской Федерации // Вестник Юридического института МИИТ. 2021. № 1 (33). С. 44-55.

20. Пономаренко Н.Н. Государственный финансовый контроль: принципы организации // Сборник научных работ серии «Финансы, учет, аудит». 2020. № 3 (19). C. 154-165.

( Турищева Татьяна Борисовна ( ttb2812@mail.ru).

Журнал «Современная наука: актуальные проблемы теории и практики» 